Nutr Metab Cardiovasc Dis. 2012 September ; 22(9): 734-740. doi:10.1016/j.numecd.2010.11.011.

\title{
The association of biomarkers of iron status with mortality in US adults
}

\author{
A Menke ${ }^{\mathrm{a}}$, P Muntner ${ }^{\mathrm{b}}$, JM Fernández-Real ${ }^{\mathrm{c}, \mathrm{d}}$, and E Guallar ${ }^{\mathrm{a}, \mathrm{e}}$ \\ a Department of Epidemiology, Johns Hopkins Bloomberg School of Public Health, Johns \\ Hopkins University, Baltimore, MD, US \\ b Department of Epidemiology, University of Alabama at Birmingham, Birmingham, AL, US \\ ' Department of Diabetes, Endocrinology, and Nutrition, Institut d'Investigació Biomédica de \\ Girona, Girona, Spain \\ d CIBEROBN Fisiopatología de la Obesidad y Nutrición, Girona, Spain \\ e Department of Cardiovascular Epidemiology and Population Genetics, National Center for \\ Cardiovascular Research (CNIC), Madrid, Spain
}

\begin{abstract}
Background and Aims-Elevated iron biomarkers are associated with diabetes and other cardiometabolic abnormalities in the general population. It is unclear whether they are associated with an increased risk of all cause or cause-specific mortality. The purpose of the current analysis was to evaluate the association of ferritin and transferrin saturation levels with all-cause, cardiovascular, and cancer mortality in the general US adult population.
\end{abstract}

Methods and Results-A prospective cohort study was conducted with 12,258 adults participating in the Third National Health and Nutrition Examination Survey (NHANES III), a nationally representative sample of the US population. Study participants were recruited in 1988 to 1994 and followed through December 31, 2006 for all-cause, cardiovascular disease, and cancer mortality. The multivariable-adjusted hazard ratios (95\% confidence interval) for all-cause mortality comparing the fourth versus the second quartiles of ferritin and transferrin saturation were $1.09(0.82-1.44$; $\mathrm{p}$-trend across quartiles $=0.92)$ and $1.08(0.82-1.43$; $\mathrm{p}$-trend across quartiles $=0.62)$, respectively, for men, $1.43(0.63-3.23$; $p$-trend across quartiles $=0.31)$ and 1.48 $(0.70-3.11 ; p$-trend across quartiles $=0.60)$, respectively, for premenopausal women, and 1.03 $(0.79-1.34 ; \mathrm{p}$-trend across quartiles $=0.95)$ and 1.17 (0.92-1.49; $\mathrm{p}$-trend across quartiles $=0.63)$, respectively, for postmenopausal women. Quartile of ferritin and transferrin saturation also showed no association between biomarkers of iron status and mortality.

Conclusions-In a large nationally representative sample of US adults, within the spectrum of normal iron metabolism, ferritin and transferrin saturation were not associated with risk of mortality among people who were not taking iron supplements and did not have a baseline history of cardiovascular disease or cancer.

\footnotetext{
Address correspondence and reprints requests to: Eliseo Guallar, Welch Center for Prevention, Epidemiology, and Clinical Research, 2024 E. Monument Street; Room 2-639, Baltimore MD, 21205, (410) 614-0574, Fax: (410) 955-0476, eguallar@jhsph.edu.

Publisher's Disclaimer: This is a PDF file of an unedited manuscript that has been accepted for publication. As a service to our customers we are providing this early version of the manuscript. The manuscript will undergo copyediting, typesetting, and review of the resulting proof before it is published in its final citable form. Please note that during the production process errors may be discovered which could affect the content, and all legal disclaimers that apply to the journal pertain.
} 


\section{Introduction}

Iron has a catalytic role in the generation of highly reactive oxygen species, such as hydroxyl radical, through Fenton and Haber-Weiss reactions [1]. As a result, elevated iron levels, below the levels found in genetic hemochromatosis, may have a role in a variety of disease processes. Body iron stores can be estimated using serum ferritin and previous studies have found that elevated ferritin levels, below the levels found in genetic hemochromatosis, are associated with diabetes, metabolic syndrome, hypertension, dyslipidemia, elevated fasting insulin and glucose, and abdominal adiposity [2-4]. The association of ferritin and other biomarkers of iron metabolism with mortality, however, remains controversial. Few studies are available investigating the association between iron biomarkers and mortality, and those studies are particularly limited in their ability to investigate this association in non-white populations [5-7].

The purpose of the current analysis was to evaluate the association of ferritin and transferrin saturation levels with all-cause, cardiovascular, and cancer mortality in the general US adult population. To do so, we analyzed data from the Third National Health and Nutrition Examination Survey (NHANES III) Mortality Study, a cohort study based on a nationally representative sample of US adults with ferritin and transferrin saturation measured in 1988 to 1994 and followed for mortality through December 31, 2006.

\section{Methods}

\section{Study Population}

NHANES III was a stratified, multistage probability survey designed to be representative of the civilian non-institutionalized US population [8]. Overall, 18,825 adults $\geq 20$ years of age completed the NHANES III interview and examination. We excluded 859 participants who were taking iron supplements, 227 pregnant women, 34 participants with likely hemochromatosis (serum iron $>190 \mathrm{ug} / \mathrm{dL}$ for men and $>175 \mathrm{ug} / \mathrm{dL}$ for women, serum ferritin $>300 \mathrm{ng} / \mathrm{mL}$ for men and $>200 \mathrm{ng} / \mathrm{mL}$ for women, and transferrin saturation $>60 \%$ ), 1347 participants with a self-reported history of cardiovascular disease at baseline, 588 participants with a self-reported history of cancer at baseline, 126 participants $\geqslant 90$ years of age, 21 participants missing follow-up information, 2156 participants missing data for ferritin, 37 missing data for transferrin saturation, 210 missing data for income, 73 missing data for education, 507 missing data for alcohol use, 20 missing data for body mass index, 19 missing data for blood pressure, 32 missing data for diabetes, 55 missing data for total cholesterol, 87 missing data for HDL-cholesterol, and 169 missing data for estimated glomerular filtration rate. A total of 12,258 NHANES III participants were thus available for the current analyses.

All participants gave written informed consent. The National Center for Health Statistics of the Centers for Disease Control and Prevention Institutional Review Board approved the protocol for NHANES III.

\section{Baseline Data Collection}

Baseline data for NHANES III were collected during an in-home interview and a subsequent visit to a mobile examination center. A detailed description of all data collection methods is a available elsewhere [8]. During the in-home interview, a standardized questionnaire was used to collect demographic and health-related information including age, race-ethnicity, and sex. Additional questionnaire data collected included education, household income, smoking status, alcohol consumption, menopause status (women who reported not menstruating in the previous 12 months were considered postmenopausal), and use of 
vitamin C supplementation, aspirin, antihypertensive, lipid lowering medication, or hormone replacement therapy (among postmenopausal women).

Weight and height were measured and body mass index (BMI) was calculated as weight in kilograms divided by height in meters squared. We averaged all blood pressure measurements including three measurements during the in-home interview and three additional measurements during the visit to the mobile examination center. Plasma glucose was measured using an enzymatic reaction and we defined diabetes mellitus as fasting plasma glucose $\geq 126 \mathrm{mg} / \mathrm{dL}$, nonfasting plasma glucose $\geq 200 \mathrm{mg} / \mathrm{dL}$, and/or a self-reported history of diabetes with concurrent use of antidiabetes medication. Total cholesterol was quantified enzymatically. High density lipoprotein (HDL) cholesterol was measured after other lipoproteins were precipitated with a polyanion/divalent cation mixture. C-reactive protein (CRP) was measured using latex-enhanced nephelometry, a low-sensitivity assay. Serum creatinine was measured using a kinetic rate Jaffe method. We determined estimated glomerular filtration rate (eGFR) using the Modification of Diet and Renal Disease equation after aligning serum creatinine concentrations with the assay used in the development of the equation $[9,10]$.

Ferritin was measured by a single-incubation two-site immunoradiometric assay (Bio-Rad Laboratories, Hercules, CA). The limit of detection was $3 \mathrm{ng} / \mathrm{mL}$ and participants with a concentration below the limit of detection $(\mathrm{n}=6)$ were assigned a value of $2 \mathrm{ng} / \mathrm{mL}$. The inter-assay coefficient of variation ranged from $2.2 \%$ to $12.1 \%$. Serum iron and total ironbinding capacity were measured by a modification of the automated AAII- 25 colorimetric methods using an Alpkem rapid flow analysis system (Alpkem, Inc, Clackamas, OR). The limits of detection for iron and total iron-binding capacity were $3 \mathrm{ug} / \mathrm{dL}$ and $1 \mathrm{ug} / \mathrm{dL}$, respectively. There were no participants with iron or total iron-binding capacity below the limits of detection. The inter-assay coefficients of variation ranged from $2.0 \%$ to $4.6 \%$ for iron and from $3.2 \%$ to $4.7 \%$ for total iron-binding capacity. Transferrin saturation was calculated as (iron/total iron-binding capacity) *100\%.

\section{Mortality Follow-Up}

NHANES III adult participants were passively followed for mortality through December 31, 2006. NHANES III participants were linked to the National Death Index to determine vital status and cause of death using probabilistic matching based on 12 identifiers (e.g., Social Security number, sex, date of birth) [11]. Identical matching methodology applied to the NHANES I Epidemiological Follow-up Study for validation purposes found that $96.1 \%$ of deceased participants and $99.4 \%$ of living participants were correctly classified. Overall, 2,133 participants died during follow-up.

The time between each study participant's NHANES III examination and the date of death, the date they turned 90 years of age, or December 31, 2006, whichever occurred first, was calculated as the follow-up for each study participant. We censored follow-up at 90 years of age since mortality was very high after this age and few participants contributed person-time experience in the $\$ 0$ years of age group.

\section{Statistical Analysis}

Ferritin and transferrin saturation levels were substantially different by gender and menopause status among women. Therefore, we conducted all analyses separately for men, premenopausal women, and postmenopausal women. We categorized ferritin and transferrin saturation levels in quartiles according to sex- and menopause-specific cut points. We calculated baseline levels of study covariates for each quartile of ferritin after adjusting for age (continuous) and race-ethnicity (non-Hispanic white/non-Hispanic black/Mexican- 
American/other) using linear regression for continuous covariates and logistic regression for dichotomous covariates.

For risk analyses, we used Cox proportional hazards regression to estimate the age and raceethnicity- and multivariable-adjusted hazards ratios and 95\% confidence intervals for allcause mortality associated with each quartile of ferritin or transferrin saturation, separately, compared with the second quartile; we used the second quartile as the reference group since participants in the first quartile might have low iron stores due to a illness or disease, possibly undiagnosed, which causes blood loss and increases mortality risk. We performed tests for linear trend across quartiles by including the median of each quartile as a continuous variable in the models. Multivariable-adjusted models included adjustment for age (continuous) and race-ethnicity (non-Hispanic white/non-Hispanic black/MexicanAmerican/other), household income $<\$ 20,000$ (yes/no), high school education (yes/no), smoking status (never/former/current), alcohol consumption (yes/no), taking vitamin C supplement (yes/no), use of aspirin (yes/no), hormone replacement therapy (among postmenopausal women; yes/no), systolic blood pressure (continuous), use of antihypertensive medication (yes/no), diabetes (yes/no), body mass index (continuous), total cholesterol (continuous), use of cholesterol-lowering medication (yes/no), HDL-cholesterol (continuous), CRP (log-transformed, continuous), and estimated glomerular filtration rate $<60 \mathrm{ml} / \mathrm{min} / 1.73 \mathrm{~m}^{2}$ (yes/no). We repeated the multivariable-adjusted analysis after further stratifying by race-ethnicity. We conducted a sensitivity analysis additionally adjusting for alanine aminotransferase (ALT) and aspartate aminotransferase (AST) in multivariableadjusted models, and the results were nearly identical (data not shown).

All data were analyzed at the National Center for Health Statistics Research Data Center using SUDAAN software (version 9.0; Research Triangle Institute, Research Triangle Park, North Carolina) to account for the complex NHANES III sampling design, including unequal probabilities of selection, oversampling, and nonresponse.

\section{Results}

The median (interquartile range) of ferritin and transferrin saturation was $142 \mathrm{ng} / \mathrm{mL}$ ( 87 $222 \mathrm{ng} / \mathrm{mL}$ ) and $28 \%$ (21-35\%), respectively, for men, $35 \mathrm{ng} / \mathrm{mL}(18-60 \mathrm{ng} / \mathrm{mL})$ and $22 \%$ (15-31\%), respectively, for premenopausal women, and $90 \mathrm{ng} / \mathrm{mL}(51-158 \mathrm{ng} / \mathrm{mL})$ and $23 \%$ (18-29\%), respectively, for postmenopausal women. Men, premenopausal women, and postmenopausal women with higher ferritin levels were more likely to be non-Hispanic black (except premenopausal women) and had a higher body mass index and total cholesterol levels; they had lower HDL-cholesterol levels, and were more likely to have diabetes mellitus and CRP $\geq 1 \mathrm{mg} / \mathrm{dL}$.

The multivariable-adjusted hazard ratios ( $95 \%$ confidence interval) for all-cause mortality comparing the fourth versus the second quartiles of ferritin and transferrin saturation were $1.09(0.82-1.44 ; \mathrm{p}$-trend across quartiles $=0.92)$ and $1.08(0.82-1.43 ; \mathrm{p}$-trend across quartiles $=0.62)$, respectively, for men, $1.43(0.63-3.23$; $p$-trend across quartiles $=0.31)$ and $1.48(0.70-3.11$; $\mathrm{p}$-trend across quartiles $=0.60)$, respectively, for premenopausal women, and 1.03 (0.79-1.34; p-trend across quartiles=0.95) and 1.17 (0.92-1.49; p-trend across quartiles $=0.63$ ), respectively, for postmenopausal women (Table 2 ). When a quadratic term was included in models, none of the resulting $p$-values were significant ( $p$-value $>0.10$ for all quadratic terms).

After stratifying by race-ethnicity, the multivariable-adjusted hazard ratios (95\% confidence interval) for all-cause mortality comparing the fourth versus the second quartiles of ferritin and transferrin saturation were $1.04(0.72-1.52)$ and $1.00(0.73-1.37)$, respectively, for non- 
Hispanic white men, $1.09(0.77-1.54)$ and $1.45(0.91-2.30)$, respectively, for non-Hispanic black men, and $1.00(0.53-1.90)$ and $0.87(0.48-1.56)$, respectively, for Mexican-American men (Table 3). The multivariable-adjusted hazard ratios (95\% confidence interval) for allcause mortality comparing the fourth versus the second quartiles of ferritin and transferrin saturation were $1.43(0.50-4.10)$ and $1.27(0.49-3.27)$, respectively, for non-Hispanic white premenopausal women, $1.66(0.61-4.51)$ and $1.15(0.40-3.36)$, respectively, for nonHispanic black premenopausal women, and $0.48(0.10-2.29)$ and $2.10(0.57-7.68)$, respectively, for Mexican-American premenopausal women. The multivariable-adjusted hazard ratios (95\% confidence interval) for all-cause mortality comparing the fourth versus the second quartiles of ferritin and transferrin saturation were $1.09(0.80-1.48)$ and 1.18 (0.88-1.59), respectively, for non-Hispanic white postmenopausal women, 1.36 (0.79-2.34) and $1.26(0.77-2.07)$, respectively, for non-Hispanic black postmenopausal women, and 1.39 (0.83-2.33) and 0.64 (0.43-0.96), respectively, for Mexican-American postmenopausal women.

\section{Discussion}

In this large, population-based prospective study, higher levels of ferritin and transferrin saturation were not associated with an increased risk of mortality among NHANES III participants without a baseline history of cardiovascular disease or cancer. Among postmenopausal women, lower levels of transferrin saturation were associated with a higher risk of mortality. This finding was not observed in men or premenopausal women, and we cannot discard the possibility that this is either a chance finding or the result of residual confounding due to undiagnosed health conditions associated with reduced iron levels and increased risk of mortality.

Our results are consistent with results from the NHANES II Mortality Study, which found no association between ferritin and all-cause mortality [6]. Conversely, in the NHANES I Epidemiologic Follow-up Study, participants with a transferrin saturation $255 \%$ had an increased risk of all-cause mortality [5]. In a separate study using the NHANES I Epidemiologic Follow-up Study data, higher quintiles of transferrin saturation were not associated with an increased risk of mortality; indeed, there was some evidence of an inverse association between transferrin saturation and all-cause mortality [12]. In a study of 260 Finnish adults, transferrin saturation was not associated with risk of mortality while total iron-binding capacity, which is negatively correlated with iron stores, was inversely associated with risk of cancer mortality among women but not men [7].

Our findings are also consistent with studies investigating iron biomarkers and coronary heart disease incidence. In a meta-analysis of 12 prospective studies including 7800 cases of coronary heart disease, no biomarkers of iron status investigated were associated with an increased risk of coronary heart disease [13]. Furthermore, our findings are consistent with a meta-analysis that failed to find an association between HFE gene mutations and cardiovascular disease risk [14]. Subjects with HFE gene mutation have life-time increased iron stores compared to the general population, and provide a Mendelian randomization approach to the evaluation of the impact of increased biomarkers of iron status on health outcomes. Although patients with symptomatic hemochromatosis are at greater risk of morbidity and mortality, HFE gene mutations are not consistently associated with classical symptoms of hemochromatosis [15]. One possible explanation is that only extremely high levels of iron stores pose a health risk.

Several studies have investigated whether phlebotomy confers health benefits due to reduced iron levels in the body, usually focusing on outcomes such as diabetes mellitus and coronary heart disease. The majority of these studies have found phlebotomy confers protection from 
diabetes mellitus and coronary heart disease [16-21]. An exception is the Health Professionals Follow-up Study in which phlebotomy was not associated with risk of diabetes, cardiovascular disease, or coronary heart disease mortality [22,23]. A randomized controlled trial including 1277 participants with PAD found that participants randomized to receive phlebotomy had a nonsignificant $15 \%$ decreased risk of mortality [24].

Several studies in recent years have shown that ferritin and transferrin saturation are associated with risk of diabetes [25-28]. This is consistent with the observation that symptomatic hemochromatosis patients have a very high prevalence of diabetes [29]. If the association between biomarkers of iron status and diabetes is a true association, then we would expect to see some association between biomarkers of iron status and risk of mortality. However, it is possible that higher iron levels result in a small increase in diabetes risk that is not strong enough to produce a significant association between biomarkers of iron status and mortality in our study.

We found that postmenopausal women in the lowest quartile of transferrin saturation had an increased risk of mortality compared to the second quartile. While this may be a chance finding, there are also several alternative explanations. Anemia is a predictor of mortality [30]. It may be a marker of illness or disease, possibly undiagnosed, which causes reduced iron stores via blood loss and increases mortality risk. Alternatively, this association may be due to residual confounding by inflammation. Inflammation causes total iron-binding capacity levels to increase, and thus transferrin saturation levels to decrease. Adjusting for low-sensitivity CRP in multivariable-adjusted models may not be adequate to control for inflammation.

Our findings need to be considered in the context of certain limitations. There may be measurement error due to within-person variability in ferritin and transferrin saturation and reliance on a single measure of the biomarkers in the current analysis. Another limitation is the lack of active follow-up of NHANES III participants. However, the reliability of mortality follow-up through the National Death Index is high (98.5\%).

Despite the limitations, our study maintained a number of strengths. NHANES III data were collected using a thorough study protocol with extensive quality control measures and technicians trained and certified in all aspects of data collection. Findings from studies using NHANES data are representative of the US noninstitutionalized civilian population. Furthermore, the large NHANES III sample size allowed us to stratify our analysis by gender and menopause status, and adjust for important confounders after excluding patients with a history of cardiovascular disease and cancer at baseline.

In a large nationally representative sample of US adults, within the spectrum of normal iron metabolism, ferritin and transferrin saturation were not associated with the risk of mortality among people who were not taking iron supplements and did not have a baseline history of cardiovascular disease or cancer. This is consistent with most previous studies investigating the effects of body iron stores on mortality and CHD risk. Future studies investigating the effect of iron on health outcomes should utilize repeated measures of biomarkers of iron status to get a better measure of long-term exposure to elevated iron levels.

\section{Supplementary Material}

Refer to Web version on PubMed Central for supplementary material. 


\section{Reference List}

1. Halliwell B, Gutteridge JM. Role of free radicals and catalytic metal ions in human disease: an overview. Methods Enzymol. 1990; 186:1-85. [PubMed: 2172697]

2. Swaminathan S, Fonseca VA, Alam MG, Shah SV. The role of iron in diabetes and its complications. Diabetes Care. 2007; 30:1926-33. [PubMed: 17429063]

3. Liu Q, Sun L, Tan Y, Wang G, Lin X, Cai L. Role of iron deficiency and overload in the pathogenesis of diabetes and diabetic complications. Curr Med Chem. 2009; 16:113-29. [PubMed: 19149565]

4. Jehn M, Clark JM, Guallar E. Serum ferritin and risk of the metabolic syndrome in U.S. adults. Diabetes Care. 2004; 27:2422-8. [PubMed: 15451911]

5. Mainous AG, Gill JM, Carek PJ. Elevated serum transferrin saturation and mortality. Ann Fam Med. 2004; 2:133-8. [PubMed: 15083853]

6. Sempos CT, Looker AC, Gillum RE, McGee DL, Vuong CV, Johnson CL. Serum ferritin and death from all causes and cardiovascular disease: the NHANES II Mortality Study. National Health and Nutrition Examination Study. Ann Epidemiol. 2000; 10:441-8. [PubMed: 11023623]

7. van Asperen I, Feskens EJ, Bowles CH, Kromhout D. Body iron stores and mortality due to cancer and ischaemic heart disease: a 17-year follow-up study of elderly men and women. Int J Epidemiol. 1995; 24:665-70. [PubMed: 8550261]

8. National Center for Health Statistics. Plan and operation of the Third National Health and Nutrition Examination Survey, 1988-94. Vital Health Stat. 1994:1.

9. Levey AS, Coresh J, Greene T, Stevens LA, Zhang YL, Hendriksen S, et al. Using standardized serum creatinine values in the modification of diet in renal disease study equation for estimating glomerular filtration rate. Ann Intern Med. 2006; 145:247-54. [PubMed: 16908915]

10. Selvin E, Manzi J, Stevens LA, Van LF, Lacher DA, Levey AS, et al. Calibration of serum creatinine in the National Health and Nutrition Examination Surveys (NHANES) 1988-1994, 1999-2004. Am J Kidney Dis. 2007; 50:918-26. [PubMed: 18037092]

11. Centers for Disease Control and Prevention. National Center for Health Statistics. The Third National Nutrition and Health Survey Linked Mortality File: Matching Methodology. Hyattsville, MD: US Department of Health and Human Services, Centers for Disease Control and Prevention; 2006.

12. Sempos CT, Looker AC, Gillum RF, Makuc DM. Body iron stores and the risk of coronary heart disease. N Engl J Med. 1994; 330:1119-24. [PubMed: 7993405]

13. Danesh J, Appleby P. Coronary heart disease and iron status: meta-analyses of prospective studies. Circulation. 1999; 99:852-4. [PubMed: 10027804]

14. van der ADL, Rovers MM, Grobbee DE, Marx JJ, Waalen J, Ellervik C, et al. Mutations in the HFE gene and cardiovascular disease risk: an individual patient data meta-analysis of 53880 subjects. Circ Cardiovasc Genet. 2008; 1:43-50. [PubMed: 20031541]

15. Waalen J, Nordestgaard BG, Beutler E. The penetrance of hereditary hemochromatosis. Best Pract Res Clin Haematol. 2005; 18:203-20. [PubMed: 15737885]

16. Bofill C, Joven J, Bages J, Vilella E, Sans T, Cavalle P, et al. Response to repeated phlebotomies in patients with non-insulin-dependent diabetes mellitus. Metabolism. 1994; 43:614-20. [PubMed: 8177050]

17. Facchini FS, Saylor KL. Effect of iron depletion on cardiovascular risk factors: studies in carbohydrate-intolerant patients. Ann N Y Acad Sci. 2002; 967:342-51. [PubMed: 12079862]

18. Fernandez-Real JM, Penarroja G, Castro A, Garcia-Bragado F, Hernandez-Aguado I, Ricart W. Blood letting in high-ferritin type 2 diabetes: effects on insulin sensitivity and beta-cell function. Diabetes. 2002; 51:1000-4. [PubMed: 11916918]

19. Fernandez-Real JM, Lopez-Bermejo A, Ricart W. Iron stores, blood donation, and insulin sensitivity and secretion. Clin Chem. 2005; 51:1201-5. [PubMed: 15976100]

20. Meyers DG, Jensen KC, Menitove JE. A historical cohort study of the effect of lowering body iron through blood donation on incident cardiac events. Transfusion. 2002; 42:1135-9. [PubMed: 12430669] 
21. Salonen JT, Tuomainen TP, Salonen R, Lakka TA, Nyyssonen K. Donation of blood is associated with reduced risk of myocardial infarction. The Kuopio Ischaemic Heart Disease Risk Factor Study. Am J Epidemiol. 1998; 148:445-51. [PubMed: 9737556]

22. Ascherio A, Rimm EB, Giovannucci E, Willett WC, Stampfer MJ. Blood donations and risk of coronary heart disease in men. Circulation. 2001; 103:52-7. [PubMed: 11136685]

23. Jiang R, Ma J, Ascherio A, Stampfer MJ, Willett WC, Hu FB. Dietary iron intake and blood donations in relation to risk of type 2 diabetes in men: a prospective cohort study. Am J Clin Nutr. 2004; 79:70-5. [PubMed: 14684399]

24. Zacharski LR, Chow BK, Howes PS, Shamayeva G, Baron JA, Dalman RL, et al. Reduction of iron stores and cardiovascular outcomes in patients with peripheral arterial disease: a randomized controlled trial. JAMA. 2007; 297:603-10. [PubMed: 17299195]

25. Forouhi NG, Harding AH, Allison M, Sandhu MS, Welch A, Luben R, et al. Elevated serum ferritin levels predict new-onset type 2 diabetes: results from the EPIC-Norfolk prospective study. Diabetologia. 2007; 50:949-56. [PubMed: 17333112]

26. Jehn ML, Guallar E, Clark JM, Couper D, Duncan BB, Ballantyne CM, et al. A prospective study of plasma ferritin level and incident diabetes: the Atherosclerosis Risk in Communities (ARIC) Study. Am J Epidemiol. 2007; 165:1047-54. [PubMed: 17284722]

27. Jiang R, Manson JE, Meigs JB, Ma J, Rifai N, Hu FB. Body iron stores in relation to risk of type 2 diabetes in apparently healthy women. JAMA. 2004; 291:711-7. [PubMed: 14871914]

28. Rajpathak SN, Wylie-Rosett J, Gunter MJ, Negassa A, Kabat GC, Rohan TE, et al. Biomarkers of body iron stores and risk of developing type 2 diabetes. Diabetes Obes Metab. 2009; 11:472-9. [PubMed: 19207293]

29. Dymock IW, Cassar J, Pyke DA, Oakley WG, Williams R. Observations on the pathogenesis, complications and treatment of diabetes in 115 cases of haemochromatosis. Am J Med. 1972; 52:203-10. [PubMed: 5058506]

30. Zakai NA, Katz R, Hirsch C, Shlipak MG, Chaves PH, Newman AB, et al. A prospective study of anemia status, hemoglobin concentration, and mortality in an elderly cohort: the Cardiovascular Health Study. Arch Intern Med. 2005; 165:2214-20. [PubMed: 16246985] 


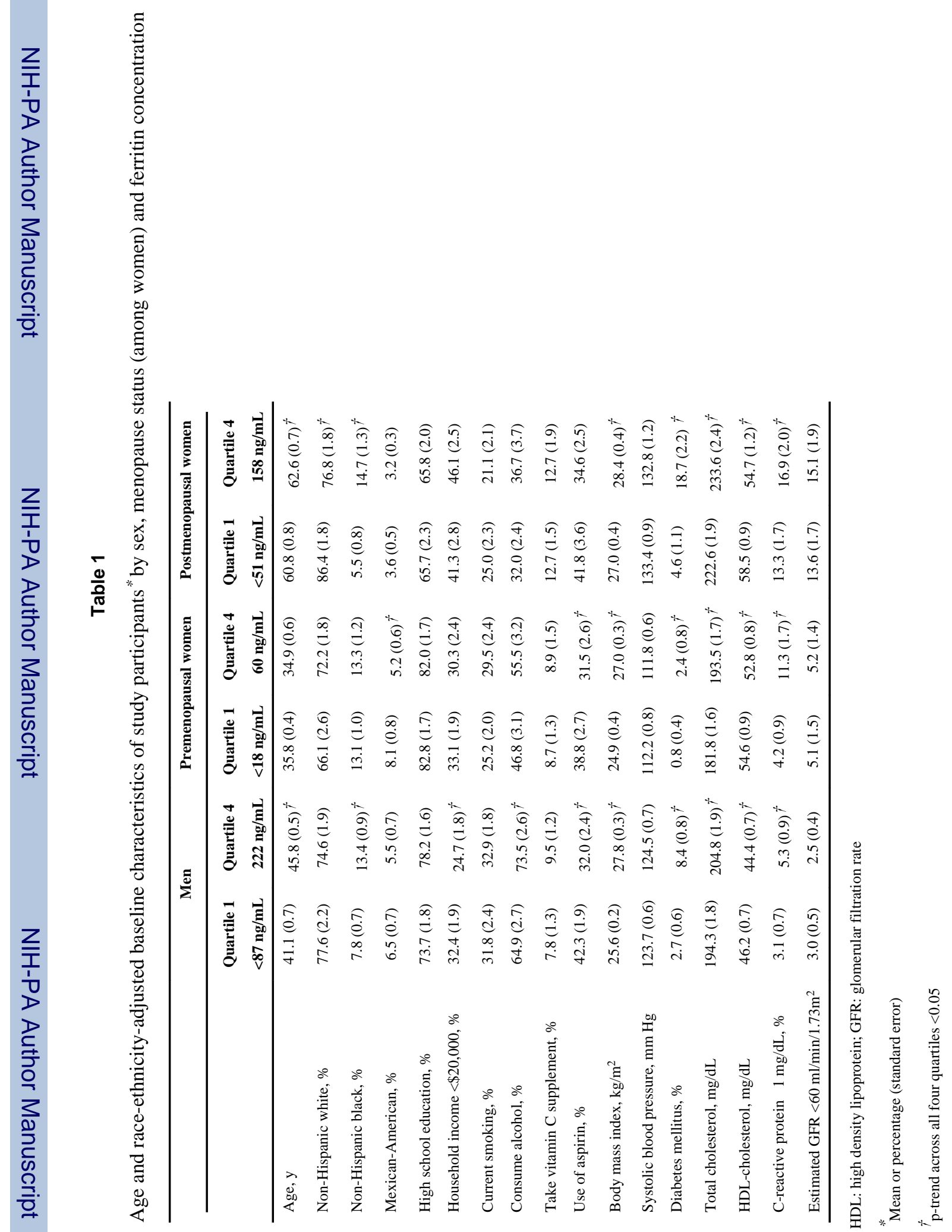




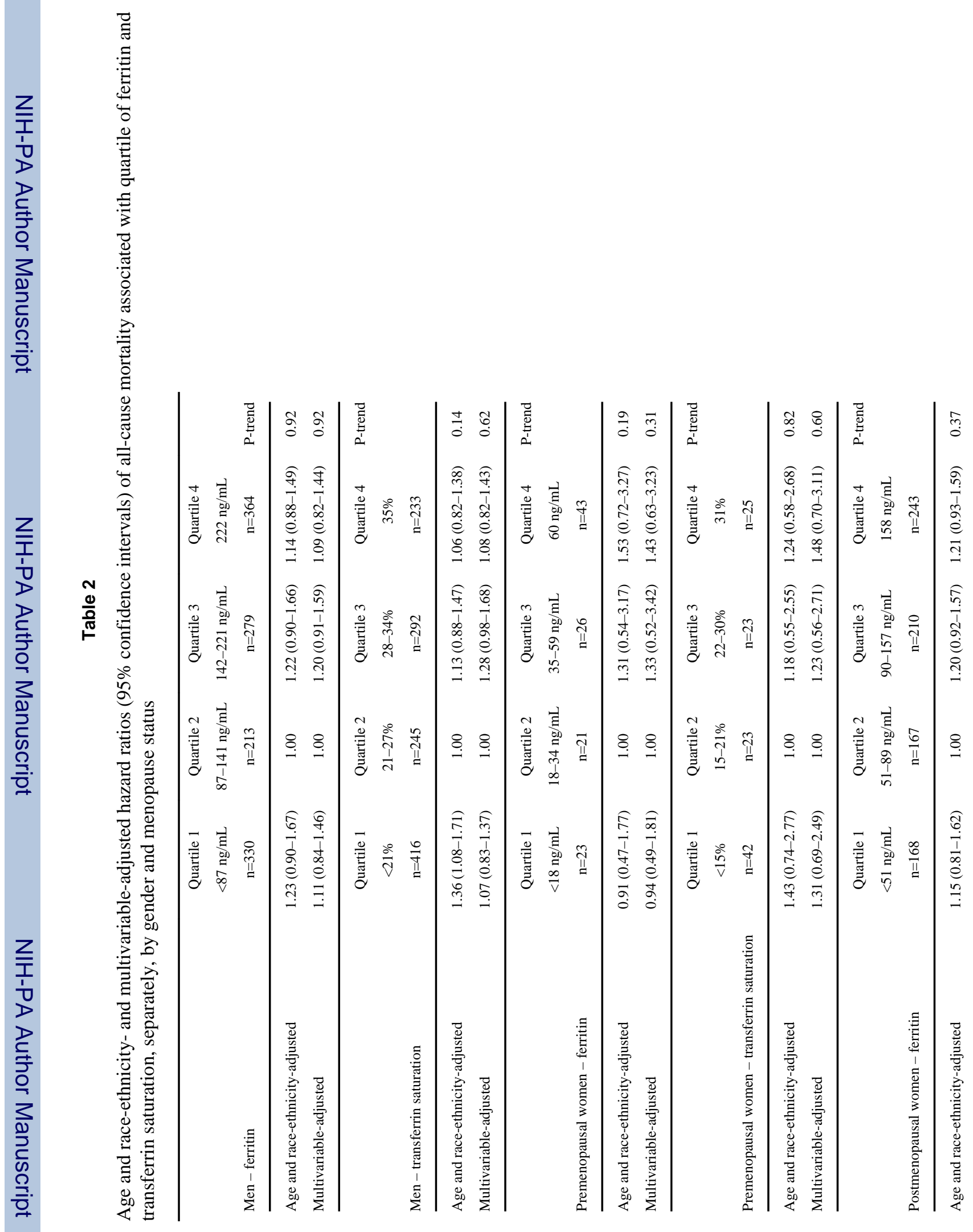




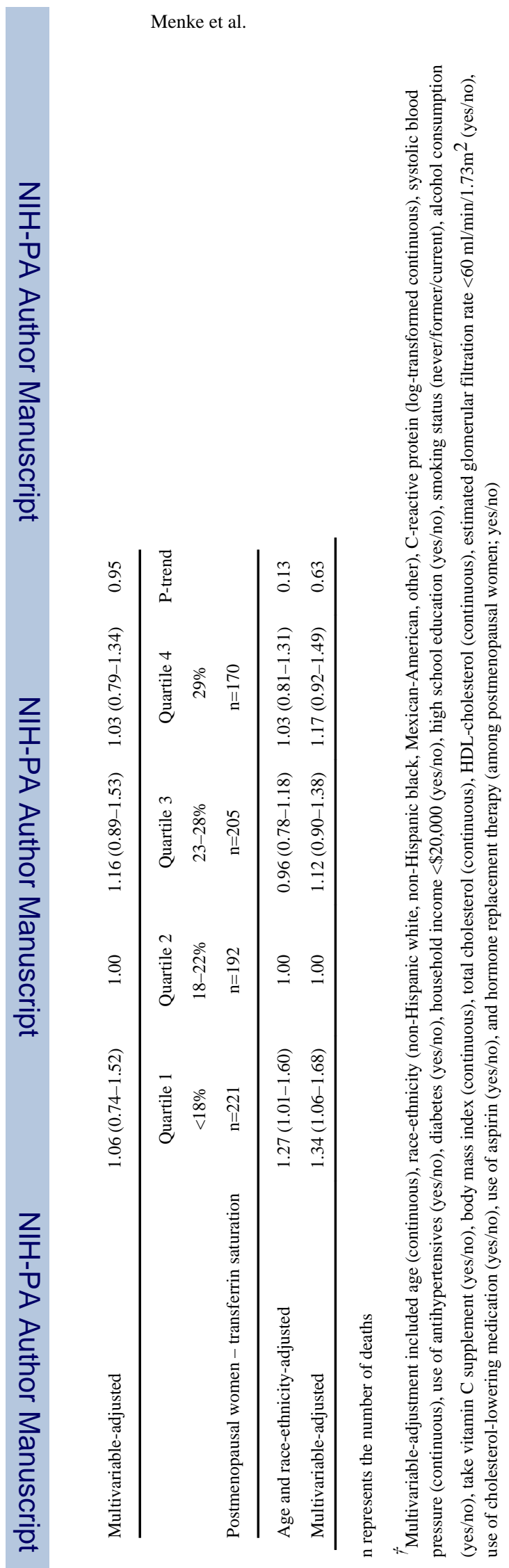

Nutr Metab Cardiovasc Dis. Author manuscript; available in PMC 2013 September 1. 


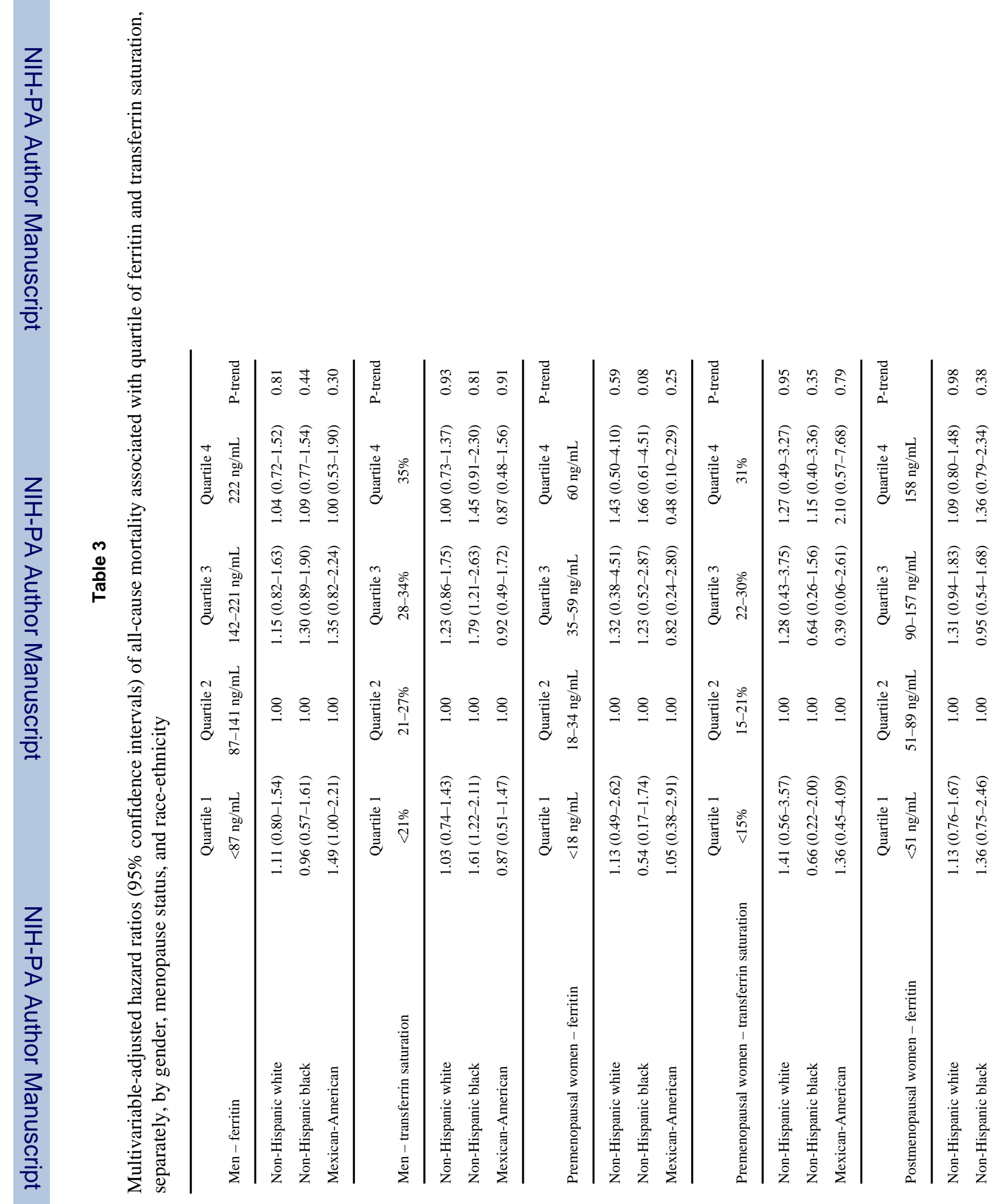




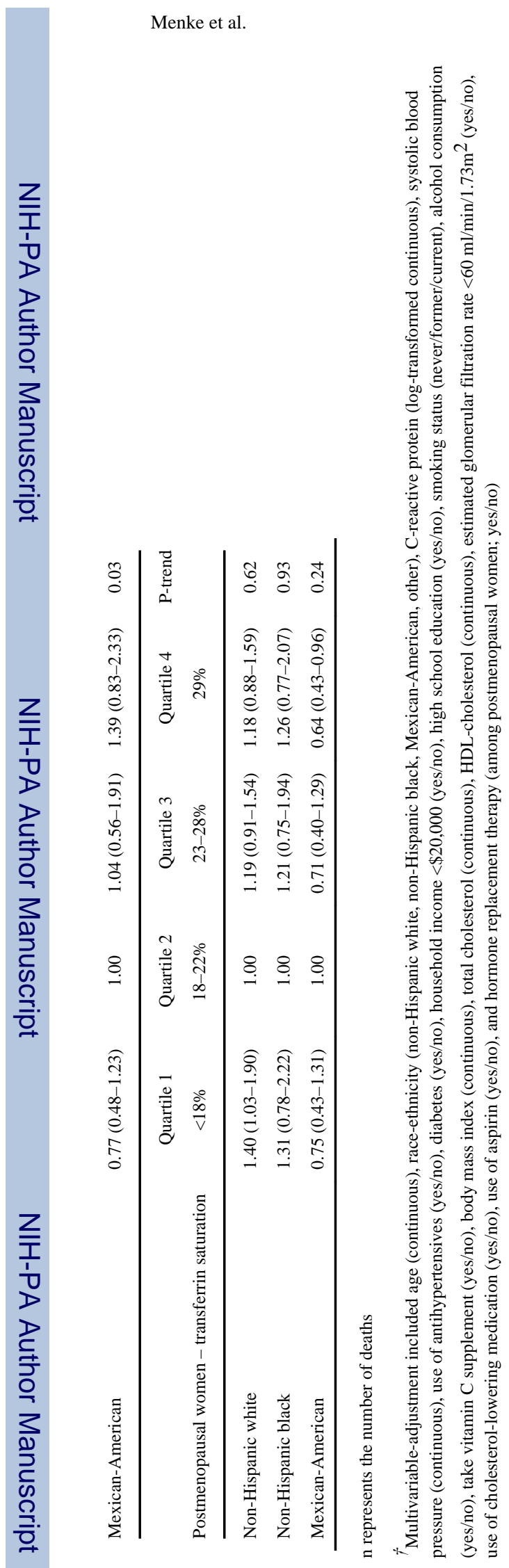

Nutr Metab Cardiovasc Dis. Author manuscript; available in PMC 2013 September 1. 